ANALYSIS OF HARDNESS AND RESISTANCE TO THE TRACTION OF AN AI-SiO 2 METAL MATRIX COMPOSITE, USING ALUMINUM CANS AND RICE HULLS AS RAW MATERIAL

\section{ANÁLISIS DE DUREZA Y RESISTENCIA A LA TRACCIÓN DE UN COMPUESTO DE MATRIZ METÁLICA AI-SiO 2 , UTILIZANDO LATAS DE ALUMINIO Y CÁSCARA DE ARROZ COMO MATERIA PRIMA}

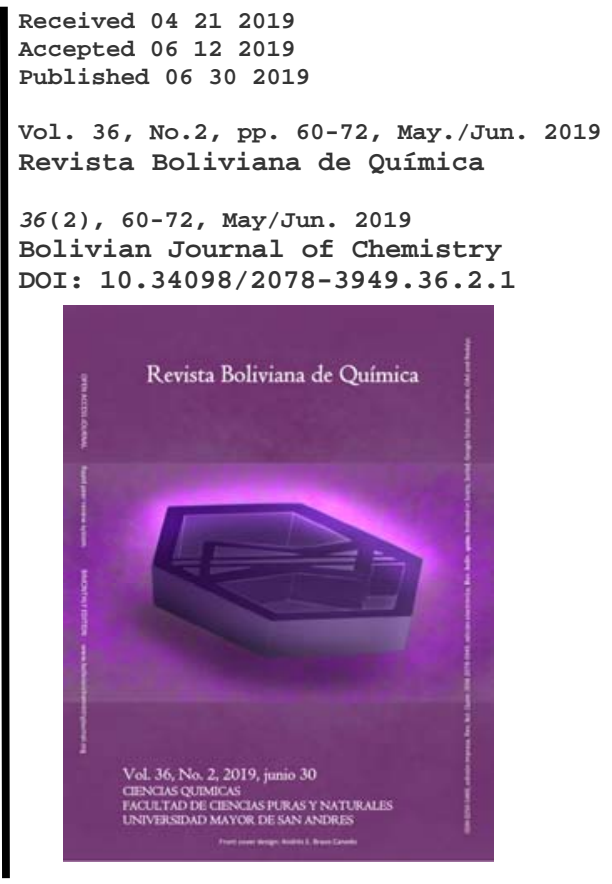

Peer-reviewed

Full original article

Cesar A. Andrade-Tacca1,*, Nemesio Edgar Veliz Llayqui², Carlos F. MedinaCaceres ${ }^{1,2}$

${ }^{1}$ Escuela Profesional de Ingenieria Ambiental, Facultad de Ingenieria de Procesos FIP, Universidad Nacional de San Agustín de Arequipa UNSA, Av. Independencia s/n-Pab. Antonio Raimondi-4to piso, Area de Ingenierias, Tel. +5154201723, Arequipa, Perú, ambiental@unsa.edu.pe, http://fip.unsa.edu.pe/ingambiental/

\begin{abstract}
${ }^{2}$ Escuela Profesional de Ingeniería Metalúrgica, Facultad de Ingeniería de Procesos FIP, Universidad Nacional de San Agustín de Arequipa UNSA, Av. Independencia s/n-Pab. Ing. Metalúrgica, Tel. +5154282813, Arequipa, Perú, metalurgia@unsa.edu.pe, http://fip.unsa.edu.pe/ingmetalurgica/
\end{abstract}

Keywords: Composite material, Aluminum, Rice hulls, Mechanical properties, Direct mixture.

Palabras clave: Material compuesto, Aluminio, Cascarilla de arroz, Propiedades mecánicas, Mezcla directa.

\title{
ABSTRACT
}

In the present investigation we have developed a metal matrix composite material (MCMM) of aluminum reinforced with rice husk ash particles (RHA).. The MCMM, with 2.5\% amorphous silica, 5\% in weight (volume), were manufactured from aluminum waste, specifically cans, and amorphous silica particles (less than $1 \mathrm{~mm}$ ) obtained from burning rice husk $(\mathrm{RH})$. The manufacturing process was based on fusion metallurgy, a direct mixing process for composite materials $(\mathrm{CM})$. In this regard, the mechanical properties of the matrix made from the can were determined, comparing them with the results of the mechanical tests of hardness and tensile strength obtained from the new MCMM.. In addition, it was determined that higher concentrations of amorphous silica in the matrix increased the hardness of the alloys, while a higher level of amorphous silica decreased elongation. 


\section{RESUMEN}

En la presente investigación hemos desarrollao un material compuesto de matriz metálica (MCMM) de aluminio reforzada con partículas de ceniza de cascarilla de arroz (CCA). Los MCMM, con sílice amorfa al 2.5\%, 5\% en peso (volumen), fueron fabricadas a partir de residuos de aluminio, específicamente latas, y partículas de sílice amorfa (menor a $1 \mathrm{~mm}$ ) obtenidas del quemado de cascarilla de arroz (CA). El proceso de elaboración se basó en la metalurgia por fusión, proceso de mezcla directa para materiales compuestos (MC). Al respecto, se determinó las propiedades mecánicas de la matriz elaborada a partir de la lata, comparándolas con los resultados de los ensayos mecánicos de dureza y resistencia a la tracción obtenidos de los nuevos MCMM. Además, se determinó que mayores concentraciones de sílice amorfa en la matriz aumentaron la dureza de las aleaciones, mientras que un mayor nivel de sílice amorfa disminuyó la elongación.

\section{INTRODUCCIÓN}

La presente investigación se centra en el estudio de las propiedades mecánicas de un MCMM de aluminio reforzada con partículas de sílice amorfa. El aluminio, hoy por hoy, está llegando a ser un sustituto ideal del acero, debido a sus propiedades de resistencia a la tracción, corrosión, ligereza, ductilidad y maleabilidad, convirtiéndolo en un material especial para las aleaciones y materiales compuestos [1-3]. Los compuestos de matriz metálica son de gran interés para aplicaciones industriales y la comparación de sus propiedades mecánicas muestran su gran impacto y utilidad [4-6].

Sin embargo, la producción de este elemento en el campo de la metalurgia, partiendo desde la extracción de bauxita y su proceso de transformación, mediante el proceso Bayer y electrólisis, genera emisiones toxicas para el medio ambiente además del consumo de una alta cantidad de energía. Debido a ello se han conducido numerosos estudios basados en el reciclado del Aluminio, las variables que afectan su ciclo de vida y los diversos aleantes y reforzantes que influyen en sus propiedades mecánicas. Con estas investigaciones se busca comprender e identificar nuevas tecnologías que incrementen el uso de aluminio secundario (aluminio desechado en botaderos), optimizando así su reciclamiento.

La eficiencia de los residuos sólidos de aluminio luego de pasar por un proceso de fundición en un horno de inducción, donde se varió la temperatura y concentración de los fundentes, se midió mediante un rendimiento del $90 \%$ [7]. Un similar trabajo realizado por Verran y Kurzawa [8] mostró un rendimiento de 93\%.

El reciclaje y aprovechamiento de las aleaciones de aluminio ha sido otro tema de interés para los investigadores ante la posibilidad de transformar los residuos en el producto original para que retornen a su aplicación inicial $[9,10]$. Un ejemplo es la aleación aluminio-silicio (Al-Si) que posee numerosas aplicaciones industriales a nivel mundial, como en el sector transporte donde aporta entre el 85 y $90 \%$ de las autopartes; gracias a su resistencia a la corrosión, resistencia al desgaste, maquinabilidad, estabilidad química y propiedades mecánicas $[11,12]$.

En Europa siete de cada 10 latas de bebidas vendidas se reciclan y el $75 \%$ del aluminio reciclado que se produce se sigue utilizando hoy en día. Además, se plantea que para el 2020 la producción de latas sobrepasaría al $65 \%$ de la producción mundial actual. Todo ello podría justificar el porque es uno de los residuos más estudiados y que más investigaciones ha motivado.

Por otro lado, la ceniza de cascarilla de arroz (CA) ha sido investigada por su alto contenido en sílice amorfa y su aplicación como reforzante en las aleaciones de aluminio [13-15]. También puede ser empleado como un potencial agente reforzante para la fabricación de compuestos de matriz metálica. Es una excelente fuente de sílice $\mathrm{SiO}_{2}$ y puede reemplazar a los recursos convencionales de dicha sal $[16,17]$.

Ante la problemática que generan las latas de aluminio desechadas en botaderos y sílice amorfa no aprovechada de la CA, en el presente trabajo se plantea reutilizar estos elementos (latas, CA) para elaborar un MCMM de aluminio- $\mathrm{SiO}_{2}$ en $2.5 \%, 5 \%$ de fracción en peso (volumen) y estudiar sus propiedades mecánicas.

\section{EXPERIMENTAL}

\section{Reactivos}


El ácido sulfúrico, fue obtenido de Sigma-Aldrich (St. Louis, USA), la antracita como combustible fue obtenida del mercado local, el fundente (coveral 11), afinador de grano (nuclenat), degasificador (Desgaser) y escorificante, obtenidos de John \& Mani India Pvt. Ltd. Chennai, (Tamil Nadu - India).

\section{Metodología}

Elaboración del material compuesto obtenido por medio de la metalurgia por fusión. A partir de extracciones de una aleación de aluminio y la fundición de las latas de aluminio; y la tostación de cascarilla de arroz, para obtener sílice amorfa. Seguidamente, el material compuesto será sometido a ensayos mecánicos, respetando las normas establecidas en los estándares ASTM para la fabricación de probetas. Finalmente se analizarán los resultados de las pruebas, comparándolas con aleaciones existentes.

\section{Proceso de fusión de latas}

En esta etapa las latas compactadas son introducidas de forma continua en el horno a una temperatura de $760^{\circ} \mathrm{C}$. Agregado del fundente para separar la escoria del metal. Agregado del escorificante para quitar la escoria de la superficie del baño líquido. Agitación constante para homogenizar el metal. Agregado de aleantes para lograr una composición química acorde a las necesidades.

Una vez obtenido el metal proveniente del proceso de reciclado de latas podemos volcar el metal líquido en un recipiente llamado lingotera y obtener una pieza de 3 kilos de peso llamado lingote, para usarlo como materia prima para su transformación posterior.

Al utilizar únicamente estos envases se garantiza, la uniformidad en la aleación obtenida, lo que permite a los futuros usuarios un mejor manejo del control de calidad del producto final

\section{Cascarilla de arroz (CA)}

La CA es un tejido vegetal constituido por celulosa y sílice, El uso de cascarilla como material abrasivo representa un aporte significativo a la preservación de los recursos naturales y un avance en el desarrollo de tecnologías limpias y económicas en la producción de arroz, uno de los principales cereales de nuestra canasta familiar [18,19]. El contenido de humedad, la composición química y el poder calorífico de la cascarilla son aspectos que hay que conocer para determinar el tipo de horno más adecuado para la quema e incineración de este subproducto agrícola [20].

Sabemos que las zonas de mayor producción de arroz están localizadas al norte del Perú, en los departamentos de Lambayeque (24\%), San Martin (16\%), La libertad (14\%) y Piura (10\%) mientras que el 21\% de la producción se encuentra en la región de Arequipa.

La CA generada en el proceso de molienda representa el $20 \%$ en peso del arroz. Actualmente, solo el $5 \%$ de la cascarilla de arroz es usado como combustible para hornos de secado de ladrillos en el departamento de Piura, el resto es quemado o arrojado a los ríos aledaños. Actualmente se generan anualmente $3039000 \mathrm{TM}$ de cascarilla de arroz que equivale a 959684.21000 TEP (Tonelada equivalente de petróleo) por año de energía que está disponible y es desperdiciada. El empleo de residuos agrícolas como combustibles es bastante restringido e ineficiente en Perú,

\section{Composición de la CA}

Es la caracterización técnica más importante y constituye la base para los análisis de los procesos y cálculos de volúmenes de aire, gases y entalpía. La composición expresa del porcentaje en masa de carbono, hidrógeno, oxígeno, nitrógeno, cenizas y humedad de la composición de la cascarilla de arroz.se muestra en la Tabla 1.

La Tabla 1 muestra los siguientes rangos de variabilidad en porcentaje para cada elemento: carbono $37.60-$ 42.60; hidrógeno 4.70 - 5.78; oxígeno 31.37 - 37.62; nitrógeno 0.38 -1.88; azufre 0.01 - 0.09; cenizas 16.93 -24.60 [20].

\section{Aplicaciones de la CA}

Las aplicaciones principales de la cenizas de cascarilla de arroz son: En vidrios, estabilización de suelos, como aislante térmico, agregado en concretos, en cemento Portland, refractarios, en materiales compuestos reforzados de chips de silicio, en purificación de agua, en caucho vulcanizado, industrias cerámicas, absorbente de aceite, 
cesar A. Andrade-Tacca et al. RBQ Vol. 36, No.2, pp.60-72, 2019

fabricación de azulejos, como polvos extinguidores de fuego, relleno abrasivo para asta dental, como clarificador de cerveza y domésticamente para limpiar productos de cristalería.

Tabla 1: Composición de la Cascarilla de arroz (CA)

\begin{tabular}{ccccccc}
\hline \multirow{2}{*}{ Parámetros } & \multicolumn{7}{c}{ VARIEDADES DE LA CASCARILLA } \\
\hline $\mathrm{C}$ & 37.60 & 42.10 & 38.70 & 42.60 & 38.83 & 37.60 \\
$\mathrm{H}$ & 5.42 & 4.98 & 4.70 & 5.10 & 4.75 & 5.78 \\
$\mathrm{O}$ & 36.56 & 33.66 & 31.70 & 33.44 & 35.47 & 37.62 \\
$\mathrm{~N}$ & 0.38 & 0.40 & 0.50 & 0.51 & 0.52 & 1.88 \\
$\mathrm{~S}$ & 0.03 & 0.02 & 0.01 & 0.02 & 0.05 & 0.09 \\
$\mathrm{CI}$ & 0.01 & 0.04 & 0.12 & 0.13 & 0.12 & 0.00 \\
CENIZA & 20.00 & 18.80 & 24.60 & 18.20 & 20.26 & 16.93 \\
TOTAL & $\mathbf{1 0 0 . 0 0}$ & $\mathbf{1 0 0 . 0 0}$ & $\mathbf{1 0 0 . 0 0}$ & $\mathbf{1 0 0 . 0 0}$ & $\mathbf{1 0 0 . 0 0}$ & $\mathbf{1 0 0 . 0 0}$ \\
\hline
\end{tabular}

Fuente: Valverde et al [20]

La metodología del presente trabajo de investigación para obtener un material compuesto reforzado con partículas de sílice amorfa y posteriormente estudiar su comportamiento mecánico, se muestra gráficamente en la Figura 1:

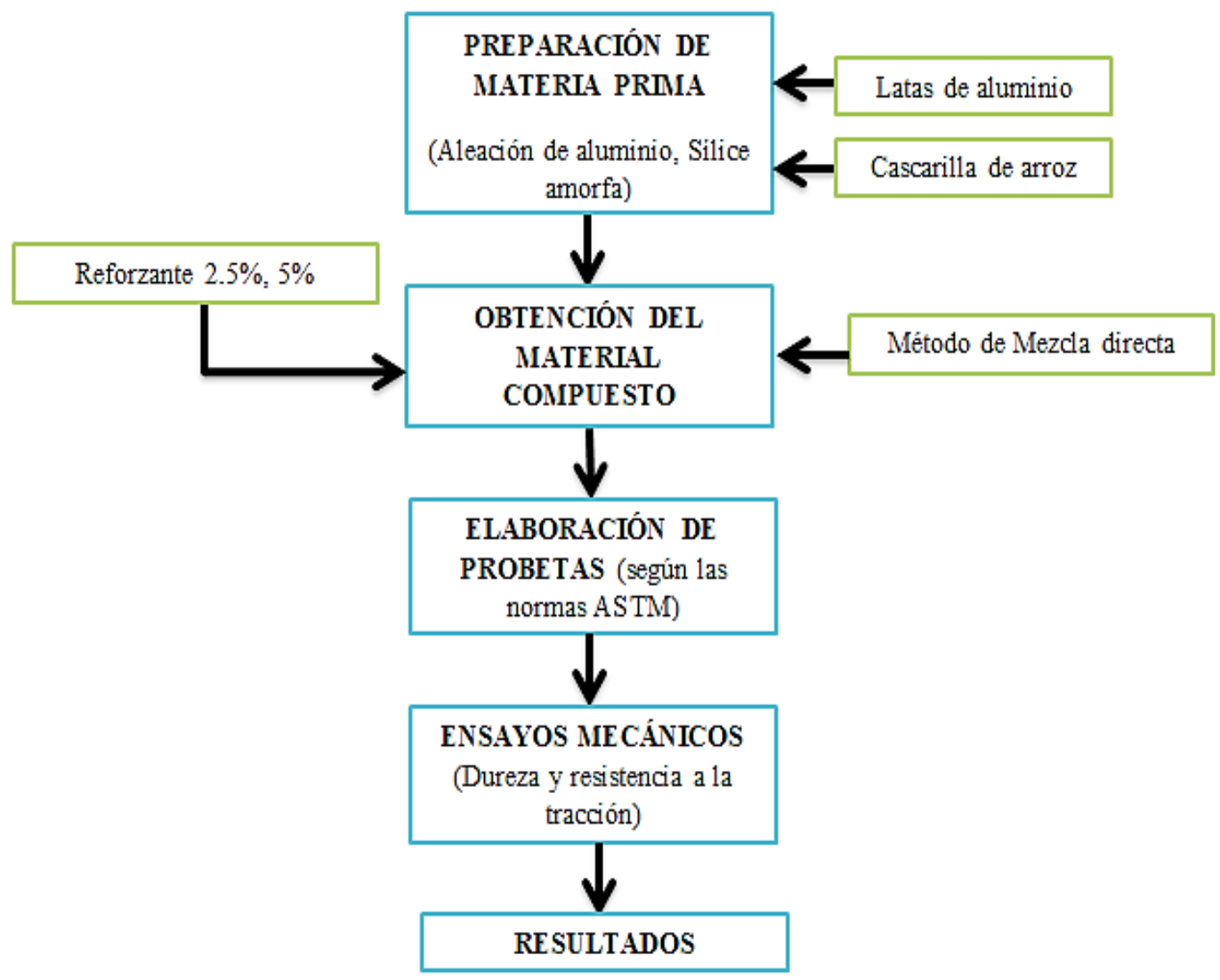

Figura 1: Diagrama de metodología de la investigación

\section{Obtención de la matriz de aluminio}


Cesar A. Andrade-Tacca et al. RBQ Vol. 36, No.2, pp. 60-72, 2019

Para obtener la matriz metálica de aluminio se procedió a preparar el horno Crisol, considerando los ladrillos refractarios y el combustible (madera y antracita). El horno se calentó al rojo vivo, temperatura mayor al punto de fusión del Aluminio $\left(660^{\circ} \mathrm{C}\right)$. Se agregó latas de aluminio, previamente compactadas, hasta completar el volumen del crisol los detalles de este proceso se observan en la Figura 2.

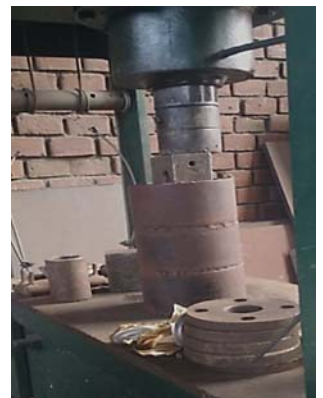

(a)

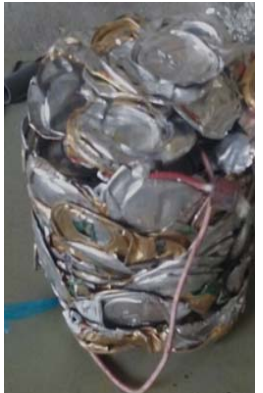

(b)

Figura 2: (a) Latas de aluminio en la compactadora (b) Latas compactadas

El proceso de colada consiste fundamentalmente en llenar un molde con la cantidad de metal fundido para obtener lingotes de un kilo como aleación matriz (Figura 3), para después de la solidificación hacer el balance químico y posteriormente obtener una aleación base.

Durante la etapa de fusión se realiza una serie de procesos para garantizar la calidad del aluminio fundido, adicionando fundentes para separar la escoria del metal, escorificador para quitar la escoria de la superficie del baño líquido, agitando para homogenizar el metal, agregando aleantes para mejorar una composición química adecuada o aleación matriz.

La colada se realizó en moldes metálicos, llamados coquillas, las cuales sirven para obtener el lingote de metal. En este método la colada se realiza vertiendo el metal fundido en un molde pre calentado. Así las piezas fundidas mostrarán una estructura de grano fino y mayor resistencia que las piezas fundidas en moldes de arena, ya que la velocidad de enfriamiento es más rápida.

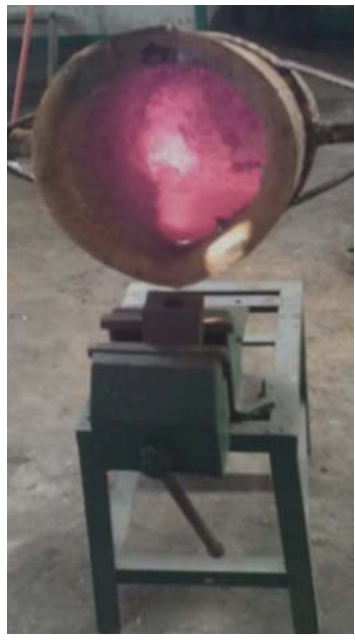

Figura 3: Colada en la coquilla

\section{Obtención de la sílice amorfa}

El procedimiento de obtención de la sílice amorfa se resume en el siguiente diagrama (Figura 4). 


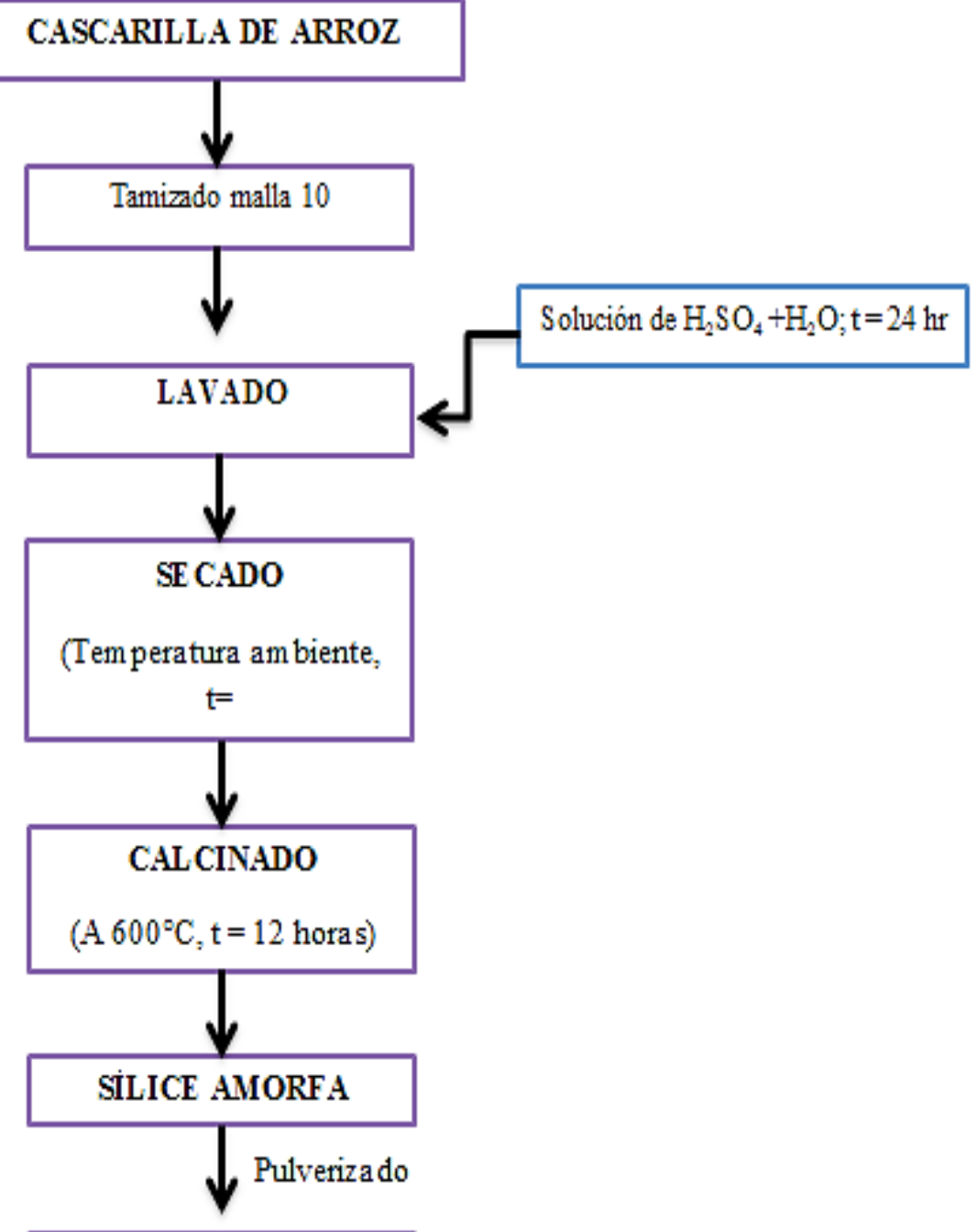

Silice a $100 \%$ malla -400

Figura 4: Diagrama de metodología para obtener CCA

Para eliminar las impurezas que contiene la CA se procede a lavarla en una solución de ácido sulfúrico $1 \mathrm{~N}$ para 12 litros de agua, durante 24 horas. Después del tiempo cumplido, con ayuda de un colador se separa la CCA de la solución y se enjuaga con agua, la cascarilla deberá ser secada durante un día.

La CA se quema a $600^{\circ} \mathrm{C}$ durante $12 \mathrm{hr}$, en un horno eléctrico. Después de esta operación, el color de la ceniza adopta un color blanco grisáceo, lo cual indica que el contenido de carbono es bajo. La ceniza con alto contenido de sílice obtenida se usa como reforzante en la elaboración de materiales compuestos en la figura 5 se observa la cascarilla de arroz al inicio y final de tratamiento.

La ceniza obtenida es llevada a la pulverizadora de anillos por 6 minutos (intervalos de 15 segundos de descanso por $1 \mathrm{~min}$ de trabajo) para obtener partículas finas $80 \%$ malla -400 .

\section{Obtención de material compuesto de matriz metálica}




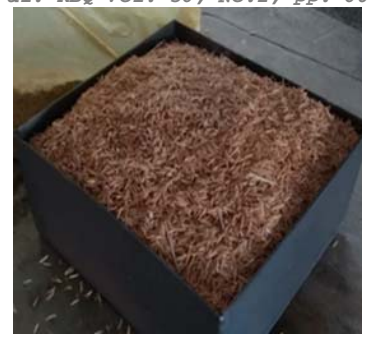

(a)

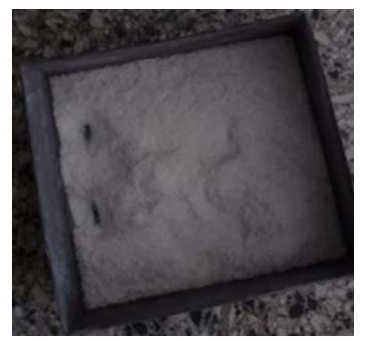

(b)

Figura 5: (a) Cascarilla de arroz

(b) Ceniza de cascarilla de arroz después de12 horas en el horno eléctrico a $600^{\circ} \mathrm{C}$

La síntesis del compuesto de matriz metálica utilizado en el presente estudio se lleva a cabo utilizando la ruta de la metalurgia líquida, mediante el proceso de mezcla directa. El proceso de fundición se llevó a cabo en un horno eléctrico con crisol de grafito de capacidad de $5 \mathrm{~kg}$, a una temperatura de fusión entre $700 \mathrm{y} 750^{\circ} \mathrm{C}$. Inicialmente, se calentó el crisol de grafito a $700{ }^{\circ} \mathrm{C}$ durante 30 minutos. Después se añadió la aleación de $\mathrm{Al}$ junto al fundente (Coveral 11), el cual reduce la oxidación y la absorción de gases. Se aumentó la temperatura a $750^{\circ} \mathrm{C}$ hasta que la aleación se fundió en el crisol. Cuando se obtuvo el baño fundido se procedió a retirar el crisol del horno e inmediatamente se vierte el afinador grano (Nucleant) y seguidamente el desgasificador (Desgaser). Un agitador de acero inoxidable se empleó en la masa fundida para homogenizar el baño fundido lentamente hasta 2/3 de la altura del metal fundido desde el fondo del crisol y el metal fundido; se agitó a una velocidad de 500 a $700 \mathrm{rpm}$, en la figura 6 se observa el momento de la agitación. Las partículas de ceniza precalentadas $(a) 600^{\circ} \mathrm{C}$ por 3 horas) se agregaron al metal fundido (ver figura 6) a una velocidad constante de 1-2 g por golpe. Para mejorar la mezcla adecuada, la velocidad del agitador aumenta gradualmente de 500 a $700 \mathrm{rpm}$ durante 4 minutos.

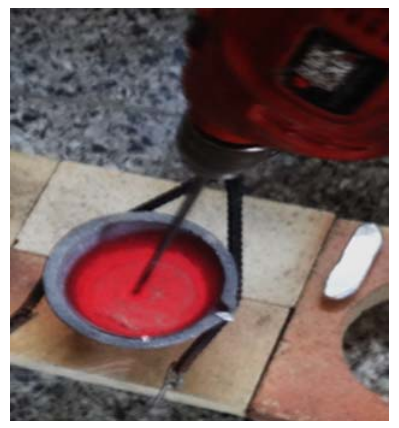

Figura 6: (a) Agitador en el baño fundido

La colada fue un molde tubular de acero inoxidable, el cual se precalentó a $500^{\circ} \mathrm{C}$ durante 30 minutos para obtener una solidificación uniforme. Se repite un procedimiento similar para la otra fracción de volumen de peso de $5 \%$ de refuerzos de partículas ceniza (sílice amorfa).

La Tabla 2 muestra las cantidades de aluminio y silicio que fueron utilizadas para la fabricación de los materiales compuestos Al-SiO2, para alcanzar una concentración de $2.5 \%$ y $5 \%$ en volumen. Por otro lado, según las hojas técnicas de los fundentes Coveral, Desgaser y Nucleant, se determinaron sus composiciones al agregarse en el baño fundido.

\section{Pruebas mecánicas}

\section{Ensayos de resistencia a la tracción}

Tabla 2: Carga (gr) a agregar para la elaboración del MCMM 


\begin{tabular}{ccccccc}
\hline Muestra & $\begin{array}{c}\text { Tamaño de } \\
\text { partícula sílice } \\
\text { amorfa }\end{array}$ & $\begin{array}{c}\text { Carga } \\
\text { aluminio (gr) }\end{array}$ & $\begin{array}{c}\text { Carga sílice } \\
\text { amorfa (gr) }\end{array}$ & $\begin{array}{c}\text { Carga } \\
\text { Coveral } \\
\text { (gr) }\end{array}$ & $\begin{array}{c}\text { Carga } \\
\text { Desgaser } \\
\text { (gr) }\end{array}$ & $\begin{array}{c}\text { Carga } \\
\text { Nucleant } \\
\text { (gr) }\end{array}$ \\
\hline Patrón & $\begin{array}{c}- \\
100 \% \text { Malla } \\
-400\end{array}$ & 300 & - & 1.5 & 1.2 & 0.6 \\
$2.5 \%$ & $\begin{array}{c}100 \% \text { Malla } \\
\mathbf{2} \%\end{array}$ & 271.6 & 2.43 & 1.3 & 1.1 & 0.54 \\
$\mathbf{5 \%}$ & 290 & 5.32 & 1.45 & 1.16 & 0.58 \\
\hline
\end{tabular}

Fuente: Elaboración propia.

Las probetas utilizadas para la prueba de tracción fueron diseñadas y mecanizadas de acuerdo a las especificaciones de la norma ASTM E8/E8M [21] para especímenes de sección circular y roscadas, la probeta de ensayo se observa en la figura 7. El equipo utilizado para esta prueba fue una maquina universal de ensayos referencia ZWICK SP600, maquina diseñada para ensayos de tracción, compresión y flexión; ubicado en los laboratorios de la Escuela de Ingeniería Mecánica de la Pontificia Universidad Católica del Perú - Lima.

Las magnitudes fundamentales que se determinaron en el equipo Zwick SP-600 son:

Esfuerzo de Fluencia, es el valor de esfuerzo que separa las zonas elásticas y plásticas para un material (en este caso aleaciones de aluminio). Un pequeño aumento en el esfuerzo por encima del límite elástico producirá un colapso en el material y provocara que se deforme permanentemente, a este comportamiento se le denomina fluencia y el esfuerzo que lo origina es el esfuerzo de fluencia.

Esfuerzo máximo, es el máximo esfuerzo registrado por las maquinas durante el ensayo de tracción, y es el esfuerzo que se produce con la máxima carga también registrada, esta carga suele ser superior a la carga ultima de rotura. Es un parámetro esencial pues refleja la capacidad resistente del material.

Elongación (\%), la máxima deformación plástica que alcanza la probeta durante el ensayo de tracción y se expresa en forma porcentual

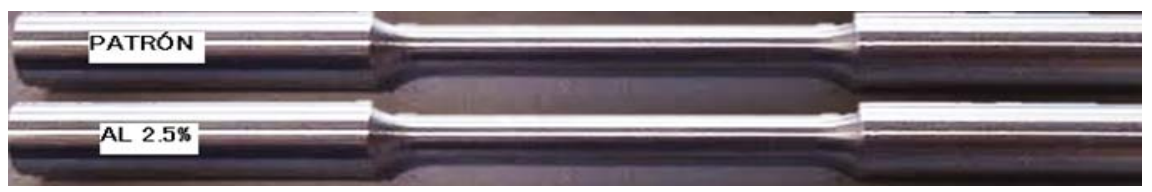

Figura 7: Probetas para el ensayo de resistencia a la Tracción

\section{Ensayos de dureza}

En el ensayo de Vickers se emplea como elemento indentador una pirámide regular de diamante, de base cuadrada, cuyas caras laterales forman un ángulo de $136^{\circ}$. En la penetración del indentador contra la probeta, éste dejará una impresión cuadrada sobre el material de la probeta, que resulta más fácil de medir (más precisa) en este tipo de ensayo. La carga que se utiliza para presionar el indentador contra la probeta oscila entre 0.001 y $120 \mathrm{Kgf}$, según la norma ASTM E-92 [22].

Por otro lado, el tiempo de aplicación de la carga durante el ensayo de Vickers oscilará entre 10 y 30 segundos, siendo 15 segundos el tiempo más empleado para la duración del ensayo. Una vez realizado el ensayo, se miden las diagonales de la impresión cuadrada que resulta sobre la superficie de la probeta ensayada y se calcula el promedio (media aritmética) de las medidas obtenidas. Para este caso se prepararon 3 pastillas de $30 \mathrm{~mm}$ de diámetro y $10 \mathrm{~mm}$ de espesor. Se utilizó el durómetro Zwick Roell ZHV $\mu$. La escala de dureza fue de VH5 y se realizaron 7 mediciones desde la superficie hacia el centro. El tiempo estimado de aplicación de la carga fue de 15 segundos.

\section{RESULTADOS Y DISCUSION}

\section{Caracterización de la matriz de aluminio}


En la Tabla 3 se observa la composición de la aleación matriz obtenida por Espectrometría de por emisión óptica (Q8 Maguellan-Bruker). La presencia de Mg y Mn se debe a la unión de las aleaciones 3004 y 5182 correspondientes a cuerpo y tapa respectivamente. Las aleaciones de la serie 3000 poseen un alto porcentaje de $\mathrm{Mn}$, mientras que las de serie 5000 presentan Mg en gran cantidad. A continuación, se muestra la composición de la matriz base.

Tabla 3: Composición de la aleación matriz

\begin{tabular}{ccccccc}
\hline Si & Fe & Mn & Mg & Zn & Al & Otros \\
\hline 0.127 & 0.451 & 0.814 & 0.670 & 0.573 & 96.7 & $1.23 \%$ \\
\hline Fuente: Pontifica Universidad Católica del Perú-2018 & & & \\
\hline
\end{tabular}

Fuente: Pontifica Universidad Católica del Perú-2018

\section{Cacterización de la ceniza de cascarilla de arroz (CCA)}

La Tabla 4 muestra la composición de la ceniza de cascarilla de arroz. El porcentaje de sílice en la ceniza obtenida, después de ser calcinada a $600^{\circ} \mathrm{C}$, es de $96.44 \%$. El punto de fusión de la sílice es mayor a $1000^{\circ} \mathrm{C}$, por lo tanto, hay una buena recuperación de sílice amorfa. La ceniza obtenida fue caracterizada para conocer su composición y el porcentaje de Sílice amorfa que presenta

Tabla 4: Composición de la ceniza de cascarilla de arroz

\begin{tabular}{ll}
\hline ELEMENTO & COMPOSICIÓN \\
\hline Oxido de calcio ( $\mathrm{CaO})$ & 0.38 \\
Oxido de Magnesio ( $\mathrm{MgO})$ & 0.13 \\
Alúmina(Al2O3) & 0.26 \\
Óxido de hierro & 1.81 \\
Oxido de manganese & 0.23 \\
Oxido de Potasio & 0.52 \\
Oxido de sodio & 0.02 \\
Sulfatos & $130 \mathrm{mg} / \mathrm{Kg}$ \\
Cloruros & $360 \mathrm{ppm}$ \\
Sílice amorfa & 96.44 \\
TOTAL & 100
\end{tabular}

Fuente: Laboratorios Analíticos del Sur, Arequipa - 2018

\section{Ensayos de dureza Vickers}

En el gráfico de la figura 8 se observa que la dureza del compuesto aumenta linealmente con el aumento en la fracción en volumen de las partículas de CCA. Esto ocurre debido a aumentos en el área superficial de la matriz y, por lo tanto, se reducen los tamaños de grano. La presencia de tal área de superficie dura ofrece más resistencia a la deformación plástica que conduce a aumentar la dureza. La relación entre el porcentaje en volumen del refuerzo de la CCA y la dureza de las CMM elaboradas se muestra en la figura 10. En la tabla 5 se puede observar las durezas de la muestra patrón y muestra con reforzantes. 


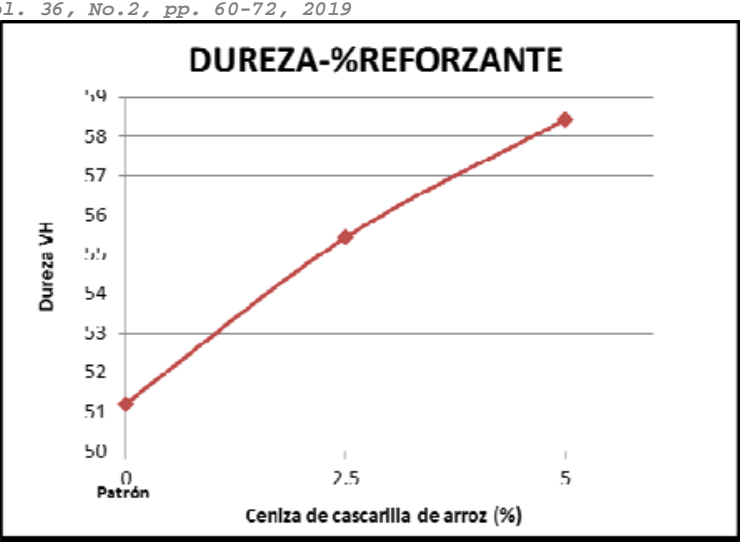

Figura 8: Diagrama de relación Dureza - \% reforzante

Tabla 5: Durezas de las muestra en escala Vickers

\begin{tabular}{cc}
\hline MUESTRA & DUREZA VH \\
\hline Patrón & 51.2 \\
Reforzante al $2.5 \%$ & 55.4 \\
Reforzante al $5 \%$ & 58.4 \\
\hline
\end{tabular}

\section{Ensayos de tracción}

A continuación, se muestra los resultados de los ensayos de resistencia a la tracción, las figuras 9, 10, 11 muestran que la máxima tensión se da a los 181.5 Mpa y en la Tabla 6 se muestran los resultados generales

Tabla 6: Resultados de los ensayos de tracción a 2.5\% y 5\% de reforzante

\begin{tabular}{lllll}
\hline Muestra & $\begin{array}{l}\boldsymbol{\sigma}_{\max } \\
(\mathbf{M P a})\end{array}$ & $\boldsymbol{\varepsilon ( \% )}$ & $\begin{array}{l}\mathbf{E} \\
(\mathbf{G P a})\end{array}$ & $\begin{array}{l}\boldsymbol{\sigma}_{\mathbf{f}} \\
(\mathbf{M P a})\end{array}$ \\
\hline Patrón & 167.8 & 5 & 39,492 & 59 \\
$2.5 \%$ & 175.6 & 3 & 53.476 & 61 \\
$5 \%$ & 181.5 & 2 & 94,745 & 90 \\
\hline
\end{tabular}

La relación entre la resistencia a la tracción de los materiales compuestos fabricados con las diferentes fracciones de volumen de partículas de ceniza de cáscara de arroz se muestra en la figura 12.

Se puede inferir que la resistencia a la tracción aumentó con un aumento en el porcentaje en peso de ceniza de cáscara de arroz. Porque, las partículas de CCA actúan como barreras a las dislocaciones cuando se toma la carga aplicada.

La mejora observada en la resistencia a la tracción del material compuesto se atribuye al hecho de que la ceniza de la cáscara de arroz de relleno posee una mayor resistencia al ofrecer más resistencia. El efecto de refuerzo por porcentaje de CCA en porcentaje de alargamiento del material compuesto se presenta en la figura 13.

En la Fig. 13 se aprecia como la ductilidad es inversamente proporcional a la dureza, se observa su disminución conforme aumenta el porcentaje de reforzante en el compuesto

\section{CONCLUSIONES}

Se concluyó que una buena dispersión de las partículas del reforzante (ceniza de cascarilla de arroz) en la matriz de aluminio, mejora la dureza y también el comportamiento mecánico. A menor tamaño de partícula del reforzante 


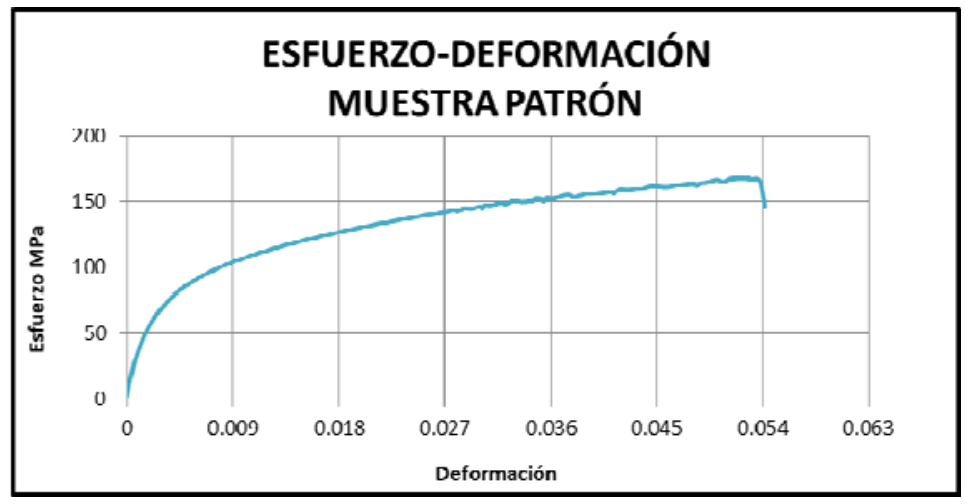

Figura 9: Gráfico de Esfuerzo - Deformación de la muestra patrón (0\% de reforzante)

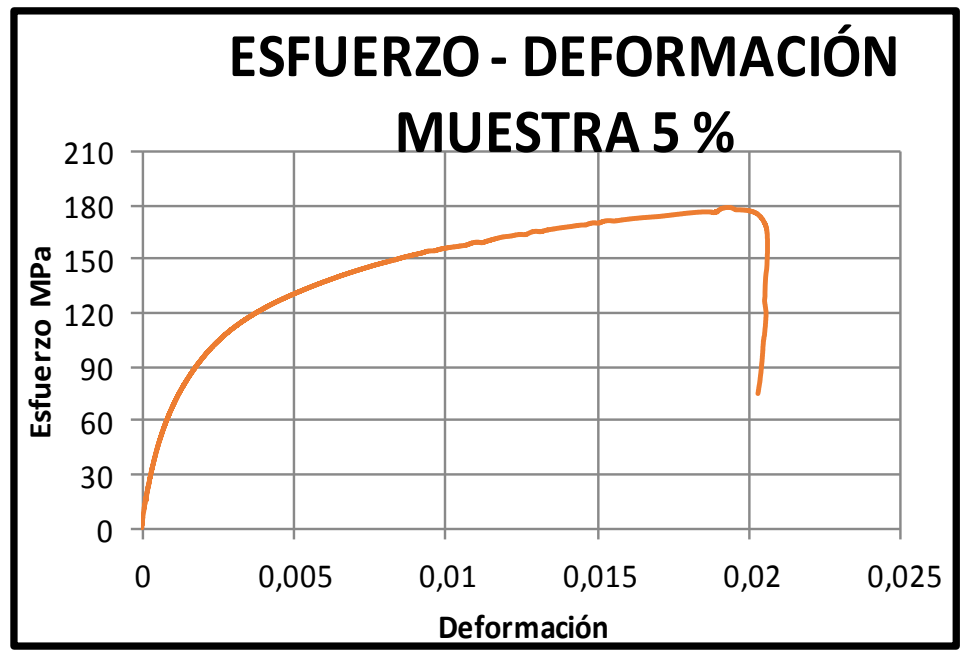

Figura 10: Gráfico de Esfuerzo - Deformación de la muestra patrón (5\% de reforzante)

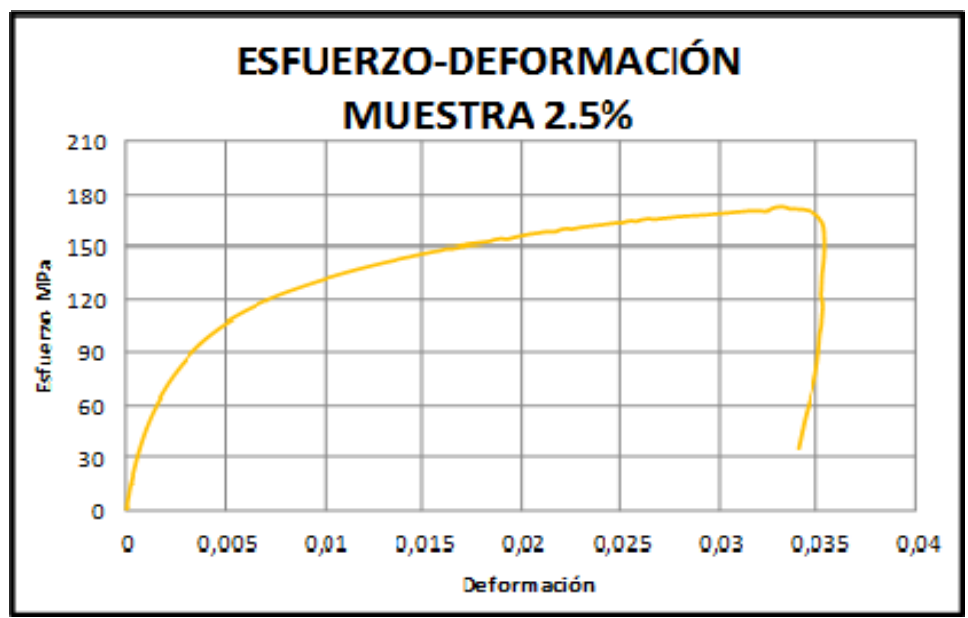

Figura 11: Gráfico de Esfuerzo - Deformación de la muestra patrón (2.5\% de reforzante) 


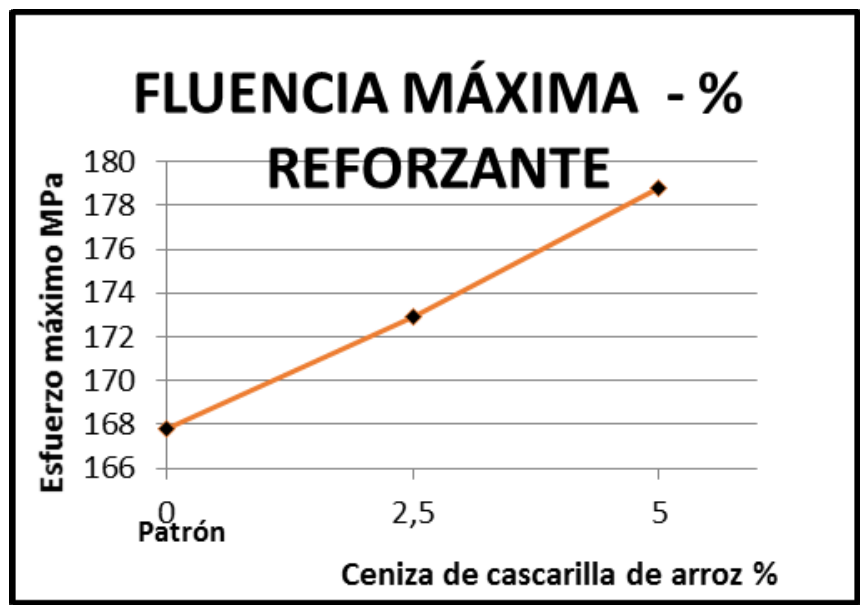

Figura 12: Gráfica de relación Fluencia máxima- \% reforzante

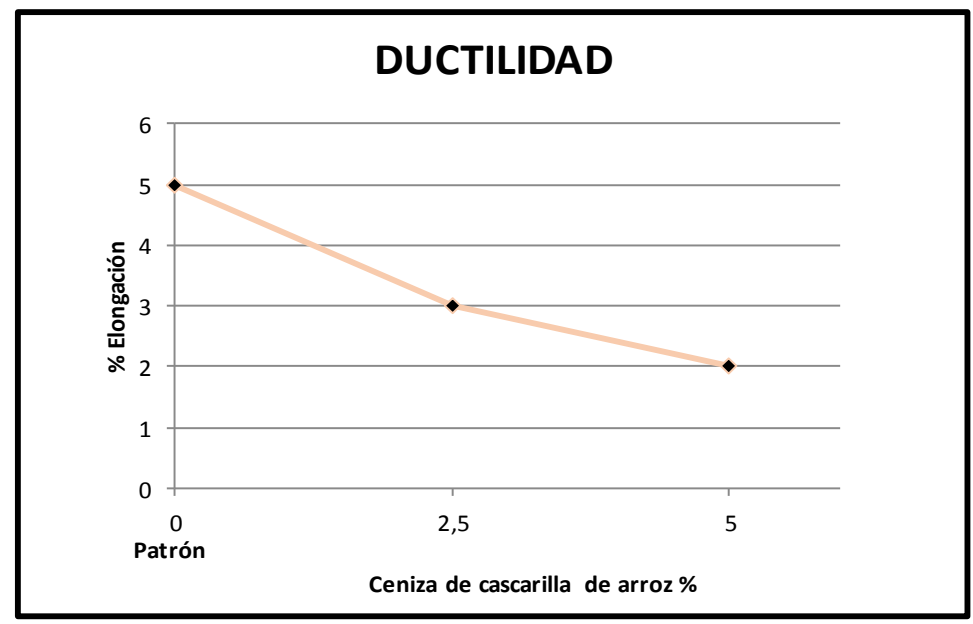

Figura 13: Relación entre el \% de elongación y el \% de reforzante

mejoran las propiedades mecánicas superando al patrón en 7.2 HV en dureza y 13.7 MPa en tracción la cual superó a las propiedades de la aleación matriz.

Se determinó que La resistencia a la tracción y la dureza aumentan con el incremento en la fracción en volumen y la ductilidad disminuye con el aumento en la fracción en volumen del reforzante

\section{RECONOCIMIENTOS}

Los autores agradecen al Vicerrectorado de Investigación de la Universidad Nacional de San Agustín de Arequipa, por el respaldo financiero.

\section{REFERENCIAS}

1. Mishra, P., Mishra, P., Rana, R.S. 2018, Effect of rice husk ash reinforcement on mechanical properties of aluminium alloy (LM6) matrix composites, Materials today: proceedings, 5, 6018-6022.

2. Vengatesh, D., Chandramohan, V. 2014, Aluminium Alloy Metal Matrix Composite: Survey Paper, International Journal of Engineering research and general Science, 2(6), 792-796.

Downloadable from: Revista Boliviana 71 de Química. Volumen 36 №2. Año 2019


3. Edil Da Costa, C., Velasco López, F., Torrealba Castello, J.M. 2000, Materiales compuestos de matriz metálica. I parte. Tipos, propiedades, aplicaciones, Revista de Metalurgia, Madrid,36,179-192.

4. Viney, K., Gupta, R.D., Batra, N.K. 2014, Comparison of Mechanical Properties and effect of sliding velocity on wear properties of Al 6061, Mg 4\%, Fly ash and Al 6061, Mg 4\%, Graphite 4\%, Fly ash Hybrid, Metal matrix composite. Procedia Materials Scienc, 6, 1365-1375.

5. Kumar Praveen, B., Birru, A.K. 2017, Microstructure and mechanical properties of aluminium metal matrix composites with addition of bamboo leaf ash by stir casting method, Transactions of nonferrous metals society of china, 27, 2555-2572.

6. Kulkarni, S.G., Meghnani, J.V., Lal, A. 2014, Effect of fly ash hybrid reinforcement on mechanical property and density of aluminium 356 alloy, Procedia Materials Science, 5, 746-754.

7. Ozer, G., Yuksel, C., Comert, Z.,Guler, K. 2013, The effects of process 103 parameters on the recycling efficiency of used aluminium beverage cans, Mater. Test. Recycl. Technol., 55, 396-400.

8. Verran, G.O., Kurzawa, U. 2008, An experimental study of aluminum can recycling using fusion in induction furnace, Resources, Conservation and recycling, 52, 731-736.

9. Tillová, E., Chalupová, M., Hurtalová L., Evolution of phases in a recycled Al-Si cast alloy during solution treatment. In: Scanning Electron Microscopy, 1st ed., 2012, V. Kazmiruk, Ed. InTech, 411-438.

10. Wang, M., Kee-do, W., Dong-keon, K., Lirong M. 2007, Study on de-coating used beverage cans with thick sulfuric acid for recycle, Energy Convers. Manag., 48, 819-825.

11. Millán Delgado, F., Sanchez Garcia, D.P., Olaya Florez J.J. 2015. Reciclaje de aluminio: Oportunidades de desarrollo en Bogota (Colombia), Gestion y Ambiente, 12(2),135-153.

12. Millán Delgado, F. 2016. Fabricación y caracterización de la aleación de aluminio reciclado con adición de silicio particulado, Tesis Universidad Nacional de Colombia. Bogotá-Colombia. Universidad Nacional de Colombia Facultad de Ingeniería, Departamento de Ingeniería Mecánica y Mecatrónica Bogotá, Colombia 2016.

13. Mejia, J.M., Mejia de Gutierrez, R., Puertas, F., 2013, Ceniza de cascarilla de arroz como fuente desílice en sistemas cementicios de ceniza volante y escoria activados alcalinamente, Materiales de construcción, 63(311), 361-375. Doi: 10.3989/mc.2013.04712.

14. Bodunrin, M.O., Kanayo Alaneme, K., Heath Chown, L. 2015, Aluminium matrix hybrid composites: a review of reinforcement philosophies; mechanical, corrosion and tribological characteristics, Journal of materials research and technology, 4, 434-445.

15. Yadav A.K., Krishna, M.P., Abhijit, D. 2018, Aluminium Metal Matrix Composite with Rice Husk as Reinforcement: A Review. Materials Today: Proceedings, 5(9), Part 3, 20130-20137.

16. Tiwari, S., Pradhan, M.K. 2017, Effect of rice husk ash on properties of aluminium alloys: A review. Materials today: proceedings, 4,486-495.

17. Serra, M.F., Conconi, M.S., Gauna, M.R., Suárez, G., Aglietti, R.F., Rendtorff, N.M. 2016, Mullite (3A12O3•2SiO2) ceramics obtained by reaction sintering of rice husk ash and alumina, phase evolution, sintering and microstructure, Journal of Asian ceramic societies, 4(1),61-67.

18. Arcos. C.A., Macías Pinto, D. , Rodríguez Páez, J.E. 2007, La cascarilla de arroz como fuente de SiO2, Revista Facultad de Ingenieria, Universidad de Antioquia.41, 7-20.

19. Vargas, J., Alvarado, P., Vega-Baudrit, J., Porras, M., 2013, Caracterización del subproducto cascarillas de arroz en búsqueda de posibles aplicaciones como materia prima en procesos, Revista científica, Facultad de Ciencias Químicas y Farmacéuticas, Universidad San Carlos de Guatemala. 23(1).

20. Valverde A.G., Sarria L.,B., Monteagudo J.P. 2007, Análisis comparativo de las características fisicoquímicas de la cascarilla de arroz, Scientia et Technica, 13(37), 255-260.

21. ASTM American Society for Testing and Materials, 2013, Standard test method for Tension Testing of Metallic Materials E8/E8M-13, USA.

22. ASTM American Society for Testing and Materials, 1997, Standard test method for Vickers Hardness of Metallic Materials E-9282 , USA. 